\title{
Raman spectral statistical classification of nasopharyngeal carcinoma and nasopharyngeal normal cell lines based on support vector classification
}

\author{
Yang Chen ${ }^{\mathrm{a}, *}$, Lei Sun ${ }^{\mathrm{a}, *}$, Yangwen Huang ${ }^{\mathrm{b}}, \mathrm{Lin} \mathrm{Ou}^{\mathrm{c}, * *}$ and Ying $\mathrm{Su}^{\mathrm{d}}$ \\ a Zhicheng College, Fuzhou University, Fuzhou, China \\ ${ }^{\mathrm{b}}$ Key Laboratory of Instrumentation Science and Dynamic Measurement Ministry of Education, North \\ University of China, Taiyuan, China \\ ${ }^{\mathrm{c}}$ College of Physics and OptoElectronics Technology, Fujian Normal University, Fuzhou, China \\ ${ }^{\mathrm{d}}$ Fujian Provincial Tumor Hospital, Fuzhou, China
}

\begin{abstract}
Raman spectroscopy (RS) has been used in the discrimination of normal and tumor cells for years. It is very important to validate an existing classification model using different algorithms. In this work, two algorithms of support vector classification (SVC) are utilized to validate our previous work about a LDA classification model of nasopharyngeal carcinoma (NPC) cell lines C666-1, CNE2 and nasopharyngeal normal cell line NP69. All of these two SVC algorithms use the same data set as the previous LDA model and, achieve great sensitivity and specificity. The final results show that our previous LDA classification model could be supported by different SVC algorithms and this demonstrates our classification model is reliable and may be helpful to the realization of RS to be one of diagnostic techniques of NPC.
\end{abstract}

Keywords: Linear discriminant analysis, nasopharyngeal carcinoma, Raman spectroscopy, support vector classification

\section{Abbreviations}

RS Raman spectroscopy;

s-p SVC self-programming SVC;

SVC support vector classification;

SVR support vector regression;

QGA quantum genetic algorithm;

LDFs linear discriminant functions.

\footnotetext{
*Yang Chen and Lei Sun are co-first authors; they contributed equally to the work.

** Corresponding author: Lin Ou, College of Physics and OptoElectronics Technology, Fujian Normal University, Building 7, Room 401, Wansheng Community (East), Shangsan Road, Cangshan District, Fuzhou, China. Tel.: +86 134 05946513; Fax: +86591 83786659; E-mail: oulin@fjnu.edu.cn.
} 


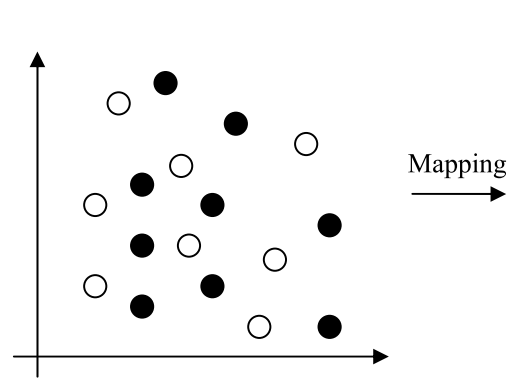

Linear undivided space

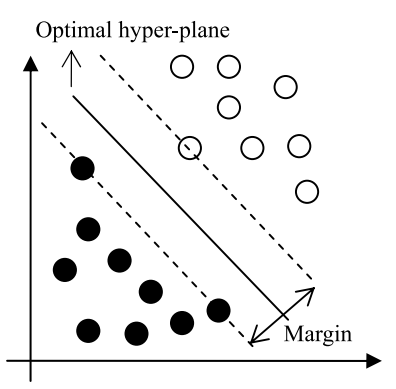

High-dimensional linear divided space

Fig. 1. The principle diagram of SVMs. The black and white circles separately represent two different classes which are maximally separated in a high-dimensional linear space.

\section{Introduction}

Nasopharyngeal carcinoma (NPC) is a malignant tumor which mainly spreads in Chinese southern area, while the incidence is relatively low in the others [6]. During the past two decades, the application of Raman spectroscopy (RS) in various fields has increased every year since an original study demonstrated the ability of RS to be an ideal optical technique [4,5,7]. Analysis of Raman spectra may provide arrays of fingerprint assignments of physical vibration mode. Such information could be used to characterize differences between human normal and tumor samples combining with various multivariate statistical analysis algorithms include partial least squares (PLS), linear discriminant analysis (LDA), support vector machines (SVMs), etc. [2,8]. However, how to evaluate a classification model's reliability is still a problem.

To our best knowledge, it is necessary to validate an existing classification model under different algorithms according to which the data set should be absolutely identical. In our previous work, a LDA model has been established to classify NPC and nasopharyngeal normal cell lines (C666-1, CNE2 and NP69) [11], and the objective of the present work is to validate this model using support vector classification (SVC). SVC is one task of SVMs which has been initially proposed by Vapnik [9]. SVMs are developed to find optimal hyper-plane constructed in a higher dimensional space according to the input data which maximal margin the two classes as shown in Fig. 1. In order to take different validations of the proposed LDA model, two SVC algorithms introduced in detail below are utilized to the validation processes.

\section{Materials and methods}

\subsection{Data sources}

All the Raman spectra of the three cell lines are acquired using a Renishaw InVia Micro-Raman system with recountal seeing [8]. Thirty, thirty-one and forty-six Raman spectra are obtained from NPC cell lines C666-1, CNE2 and normal nasopharyngeal cell line NP69, respectively. All the Raman spectra are extracted out of background autofluorescence (Vancouver Raman Algorithm, BC Cancer Agency and University of British Columbia [12]), averaged and finally normalized according to the spectral area. Then SVC is used to carry out the training and recognition. 


\subsection{SVC algorithms for the data analysis}

There are two SVC algorithms used here: one is LIBSVM (Machine Learning and Data Mining Group [3]). LIBSVM is integrated with support vector classification (C-SVC, nu-SVC), regression (epsilon-SVR, nu-SVR) and distribution estimation (one-class SVM). And what is important is that it supports multi-class classification while common SVMs always solve only a two-class classification. This matches our model well since the model is a three-class classification. Moreover, the other SVC algorithm is a self-programming SVC (s-p SVC). The basic idea of our software for a three-class classification is to break it down into several two-class problems, and then use quantum genetic algorithm (QGA) to optimize the parameters of kernel function and error penalty factor because of its high convergence rate and strong global optimization. The training algorithm is sequential minimal optimization (SMO) since it takes full advantages of the decomposition algorithm and simultaneously minimizes the size of working set so as to avoid the complex optimization process, with more details seeing [10].

\subsection{Statistical analysis}

The LDA classification model in our previous work is established on basis of 10 spectra each from C666-1, CNE2 and NP69 cell lines, respectively, which are randomly excluded to be used later as a validation set, and the remainders are used as a training set for the LDA algorithm. Once trained, the validation spectra are put into the LDA model to recognize unknown spectra. Now, for SVC in the two ways above-mentioned, the training data set is exactly the same as LDA model and the remainders are also used as testing set in the two SVC algorithms according to their respective operations.

\subsection{Estimation of model's reliability}

The classification reliability of these algorithms is estimated using error of five-fold cross-validation (E) defined by:

$$
E=\frac{N_{\text {false }}}{N_{\text {total }}}=\frac{N_{\text {total }}-N_{\text {true }}}{N_{\text {total }}},
$$

where $N_{\text {false }}, N_{\text {true }}$ and $N_{\text {total }}$ represent the number of wrongly, rightly and total classified samples, respectively [1], since five-fold cross-validation is usually used to evaluate the reliability of classification model.

\section{Results and discussion}

The scatter plot of the coefficient of each sample to classify according to the linear discriminant functions (LDFs) in LDA algorithm is given in [8] (see Fig. 2). There are two LDFs in our classification model. From this plot, C666-1, CNE2 and NP69 could be easily separated into three clusters along with the directions of LDF1 and LDF2. The classification results are depicted in Table 1. There is only one sample misjudged for every cell line, and thus $E$ is low and approximately equal. Since C666-1 and CNE2 have one sample misjudged to each other however, they are still abnormal, while one NP69 sample is misjudged to C666-1, which infect the specificity finally to $90 \%$ (sensitivity and specificity of 100 and $90 \%$, respectively shown in Table 2 ). 


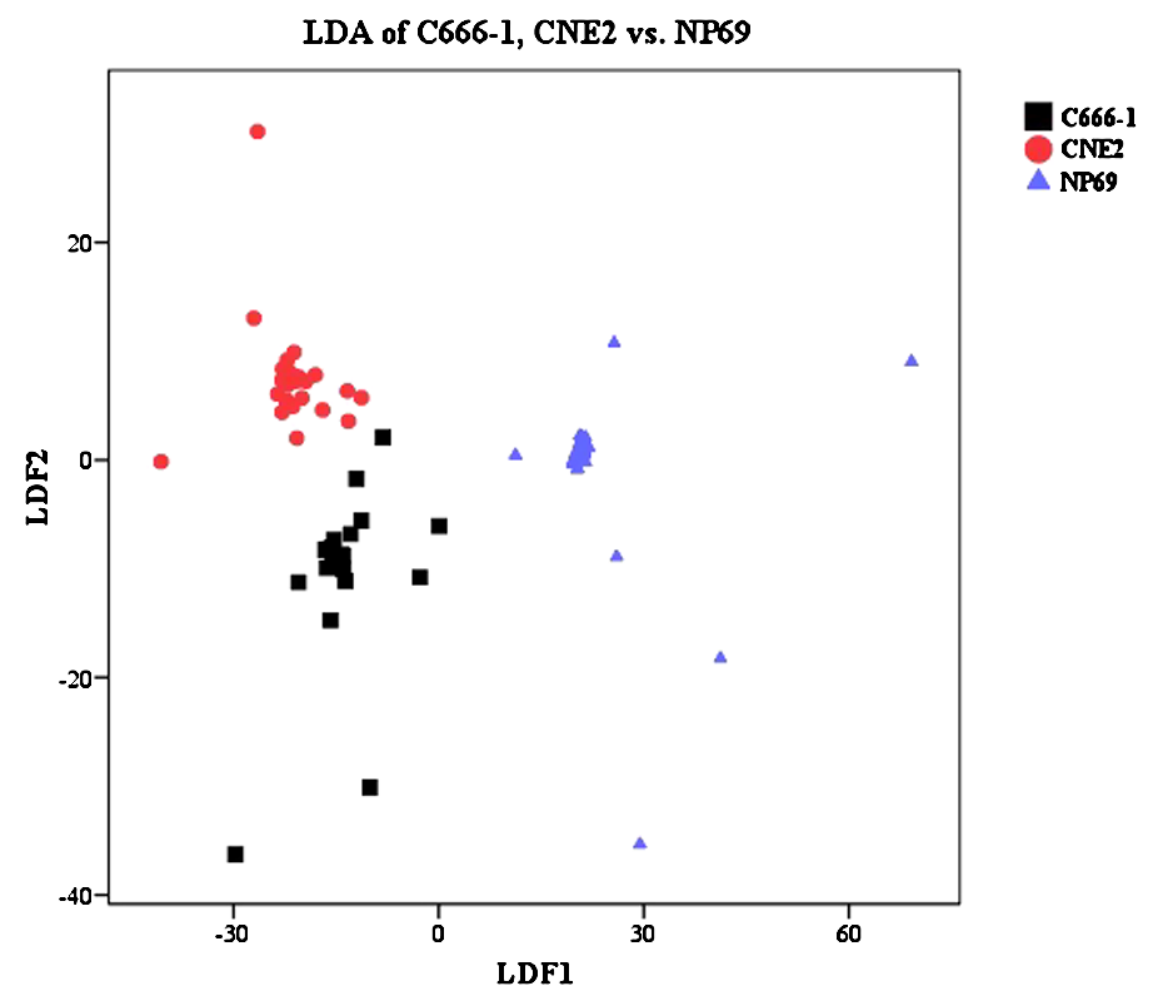

Fig. 2. LDFs' coefficient scatter plot of the LDA classification model. NPC cell lines (C666-1, CNE2) and nasopharyngeal normal cell line (NP69) could be separated into two classes along with LDF1, while C666-1 and CNE2 are separated into two classes along with LDF2. (Colors are visible in the online version of the article; http://dx.doi.org/10.3233/SPE-2011-0548.)

Table 1

Classification by LDA model: the columns show the true class while the rows refer to the tested class

\begin{tabular}{lcccccc}
\hline Cell line & $N_{\text {train }} / N_{\text {test }^{\mathrm{a}}}$ & C666-1 & CNE2 & NP69 & Total & $E(\%)^{\mathrm{b}}$ \\
\hline C666-1 & $20 / 10$ & 9 & 1 & 0 & 30 & 3.33 \\
CNE2 & $21 / 10$ & 1 & 9 & 0 & 31 & 3.23 \\
NP69 & $36 / 10$ & 1 & 0 & 9 & 46 & 2.17 \\
\hline
\end{tabular}

${ }^{\mathrm{a}} N_{\text {train }}$ - number of training samples; $N_{\text {test }}$ - number of testing samples. ${ }^{\mathrm{b}} E$ - error of five-fold cross-validation.

Table 2

The sensitivity and specificity of classification results executed by the three algorithms

\begin{tabular}{lcc}
\hline Classification algorithm & Sensitivity (\%) & Specificity $(\%)$ \\
\hline LDA & 100 & 90 \\
LIBSVM & 100 & 100 \\
s-p SVC & 100 & 90 \\
\hline
\end{tabular}

Furthermore, in the process of executing SVC of LIBSVM and the s-p SVC, the models also includes training and testing data set which are the same as the training set and unknown spectra, respectively. The SVM type is $\mathrm{C}_{\mathrm{SVC}}$ with kernel function of RBF. For LIBSVM, the automatic optimization parameter is utilized to predict the unknown samples; then for the s-p SVC, once trained, the validation spectra are put 
Table 3

Classification by SVC model of LIBSVM: the columns show the true class while the rows refer to the tested class

\begin{tabular}{lcccccc}
\hline Cell line & $N_{\text {train }} / N_{\text {test }^{\mathrm{a}}}$ & C666-1 & CNE2 & NP69 & Total & $E(\%)^{\mathrm{b}}$ \\
\hline C666-1 & $20 / 10$ & 10 & 0 & 0 & 30 & 0 \\
CNE2 & $21 / 10$ & 0 & 10 & 0 & 31 & 0 \\
NP69 & $36 / 10$ & 0 & 0 & 10 & 46 & 0
\end{tabular}

${ }^{\mathrm{a}} N_{\text {train }}-$ number of training samples; $N_{\text {test }}$ - number of testing samples. ${ }^{\mathrm{b}} E$ - error of five-fold cross-validation.

Table 4

Classification by s-p SVC model: the columns show the true class while the rows refer to the tested class

\begin{tabular}{lcccccc}
\hline Cell line & $N_{\text {train }} / N_{\text {test }^{\mathrm{a}}}$ & C666-1 & CNE2 & NP69 & Total & $E(\%)^{\mathrm{b}}$ \\
\hline C666-1 & $20 / 10$ & 10 & 0 & 0 & 30 & 0 \\
CNE2 & $21 / 10$ & 0 & 10 & 0 & 31 & 0 \\
NP69 & $36 / 10$ & 1 & 0 & 9 & 46 & 2.17 \\
\hline
\end{tabular}

${ }^{\mathrm{a}} N_{\text {train }}$ - number of training samples; $N_{\text {test }}$ - number of testing samples. ${ }^{\mathrm{b}} E$ - error of five-fold cross-validation.

into the software to be classified. Tables 3 and 4 show the classification results of these two algorithms with sensitivity and specificity representing in Table 2 .

According to Table 2, it shows that high sensitivity and specificity could be achieved by models of the SVC of LIBSVM and the s-p SVC. For LIBSVM, both the sensitivity and specificity are $100 \%$ demonstrating that no samples are misjudged; while for the s-p SVC, the misjudgments appear at NP69 where only one NP69 sample is misjudged to C666-1. On account of the identical data set of these three algorithms, such results indicate that our previous LDA classification model could be supported by kinds of SVC algorithms and act well with great sensitivity and specificity and, very interesting, the NP69 sample misjudged is the same sample in LDA and s-p SVC models attributed to the poor quality of the spectra according to our careful comparison. However, through comparison of $E$ of these three classification models, shown in Fig. 3, the accuracy $(1-E)$ of the previous LDA classification model is relatively low while high by models of the SVC of LIBSVM and the s-p SVC. Such phenomenon may indicate that no matter which SVC algorithm, the SVC of LIBSVM or the s-p SVC, is more predominant than LDA algorithm as our classification model about NPC cell lines C666-1, CNE2 and nasopharyngeal normal cell line NP69. And comparing with the SVC of LIBSVM, it can be seen that our s-p SVC algorithm should make some improvement in the ability of classifying samples with poor spectral quality. Anyway, our work is consistent with the existing results in the previous work and achieves the validation of the LDA classification model with great reliability.

\section{Conclusions}

Although RS combining with various multivariate statistical analysis algorithms is feasible to distinguish normal and tumor cells, to validate an existing classification model under different algorithms is very important for its reliability. In this work, two kinds of SVC algorithms are utilized to classify NPC cell lines C666-1, CNE2 and nasopharyngeal normal cell line NP69 with an absolutely identical data set like the previous LDA classification model. The results indicate that our previous LDA classification model could be supported by kinds of classification algorithms with great sensitivity and specificity and achieve validation of the previous LDA model with great reliability. Though these preliminary results are promising, the next work should pay more attention to make some improvement in the ability of 


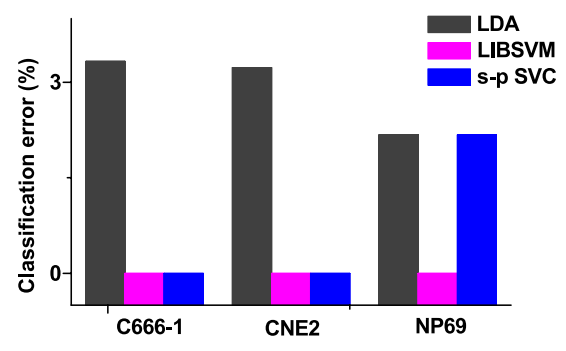

Fig. 3. Comparison of $E$ using different classification algorithms. Different accuracy is found between not only different cell lines but different classification models. And furthermore, models of the SVC of LIBSVM and the s-p SVC are more reliable than LDA classification model. $E$ - error of five-fold cross-validation. (Colors are visible in the online version of the article; http://dx.doi.org/10.3233/SPE-2011-0548.)

classifying samples with poor spectral quality, and in order to fully assess the potential of the classification model for NPC diagnosis, comprehensive knowledge of more RS of other NPC and nasopharyngeal normal cell lines should be investigated to accurately assess the diagnostic information contained in any bias of the measurements.

\section{Acknowledgements}

This work was supported by the Project of Fujian Natural Science Foundation (No. 2011J01153), and the Project of Fujian Education Department (type A, No. JA11291). Rong Chen (Fujian Normal University, Fuzhou, China), Yuhuang Ye (Fuzhou University, Fuzhou, China) are thanked for constructive discussions on the work.

\section{References}

[1] R.M. Balabin, R.Z. Safieva and E.I. Lomakina, Analytica Chimica Acta 671 (2010), 27-35.

[2] K. Bensalah et al., European Urology 58 (2010), 602-608.

[3] C.-C. Chang and C.-J. Lin, LIBSVM: a library for support vector machines, ACM Transactions on Intelligent Systems and Technology 2(27) (2011), 1-27. Software available at: http://www.csie.ntu.edu.tw/ cjlin/libsvm.

[4] S. Feng et al., Biosensors and Bioelectronics 25 (2010), 2414-2419.

[5] S. Feng et al., Biosonsers and Bioelectronics 26 (2011), 3167-3174.

[6] D.M. Parkin, S.L. Whelan, J. Ferlay, L. Teppo and D. Thomas, Cancer Incidence in Five Continents, Vol. VIII, IARC Scientific Publication No. 155, IARC Press, Lyon, 2003.

[7] G.J. Puppels, F.F.M. de Mul et al., Nature 347 (1990), 301-303.

[8] U. Schmid et al., Chemometrics and Intelligent Laboratory Systems 96 (2009), 159-171.

[9] V.N. Vapnik, The Nature of Statistical Learning Theory, Springer-Verlag, Berlin, 1995.

[10] H. Wang, Y. Huang and H. Ding, Application of support vector machine and quantum genetic algorithm in infrared target recognition, in: IPPRIE2010.

[11] Y. Ye et al., Spectroscopy 25 (2011), 217-224.

[12] J. Zhao, H. Lui, D.I. Mclean and H. Zeng, Applied Spectroscopy 61 (2007), 1225-1232. 


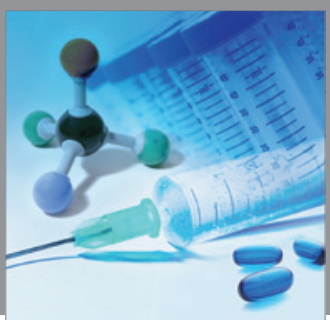

International Journal of

Medicinal Chemistry

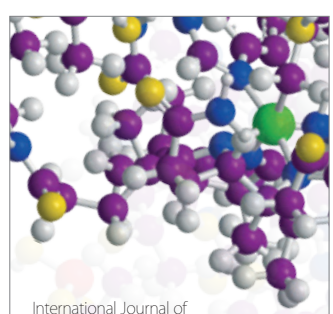

Carbohydrate Chemistry

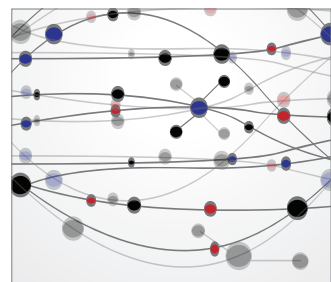

The Scientific World Journal
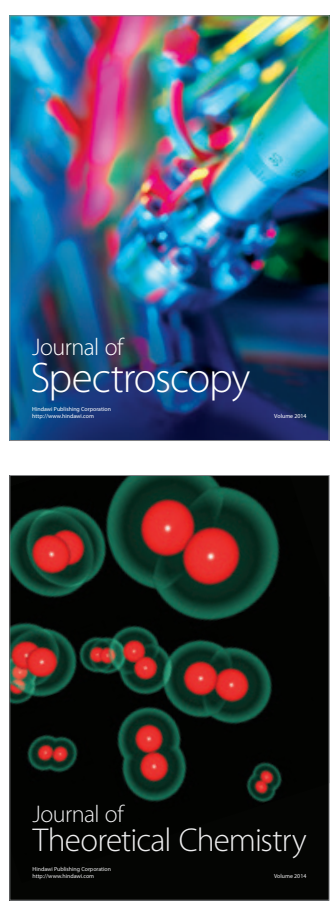
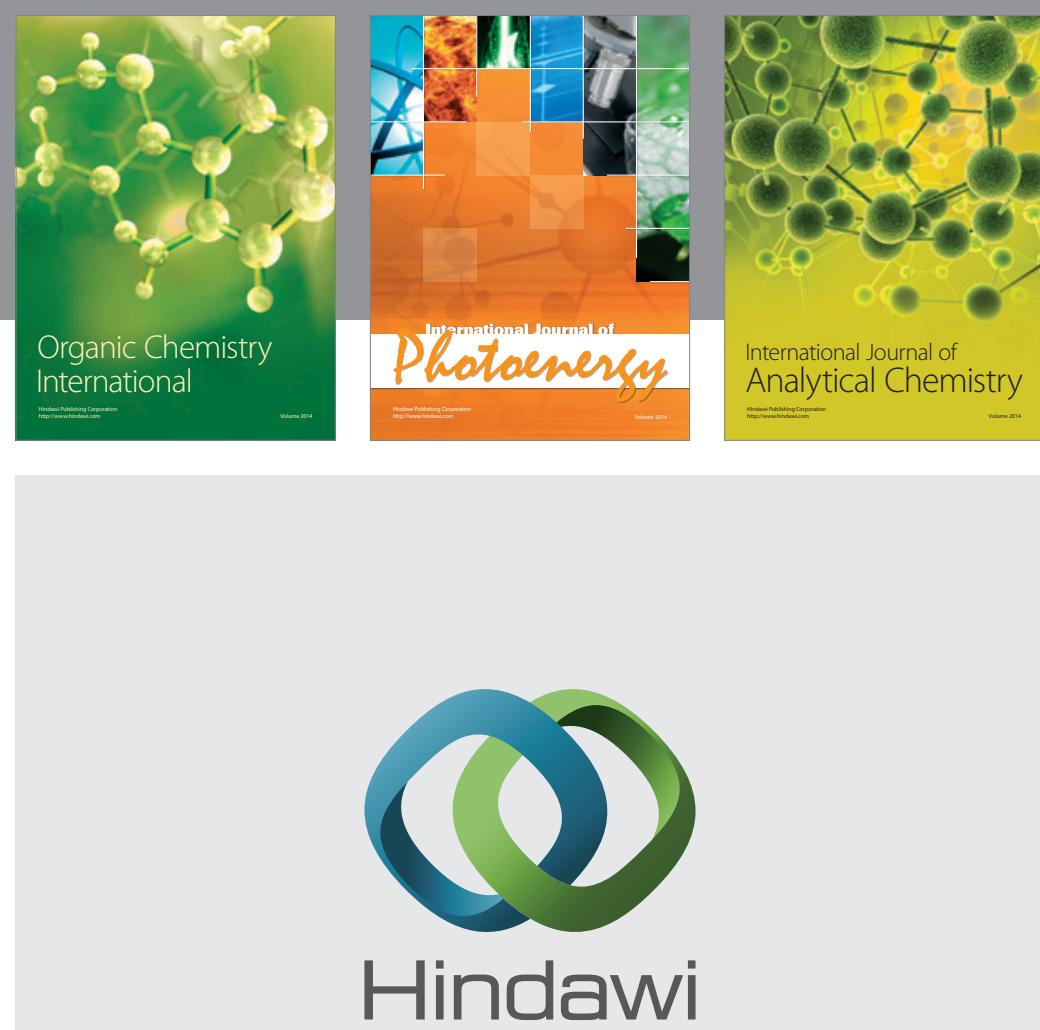

Submit your manuscripts at

http://www.hindawi.com
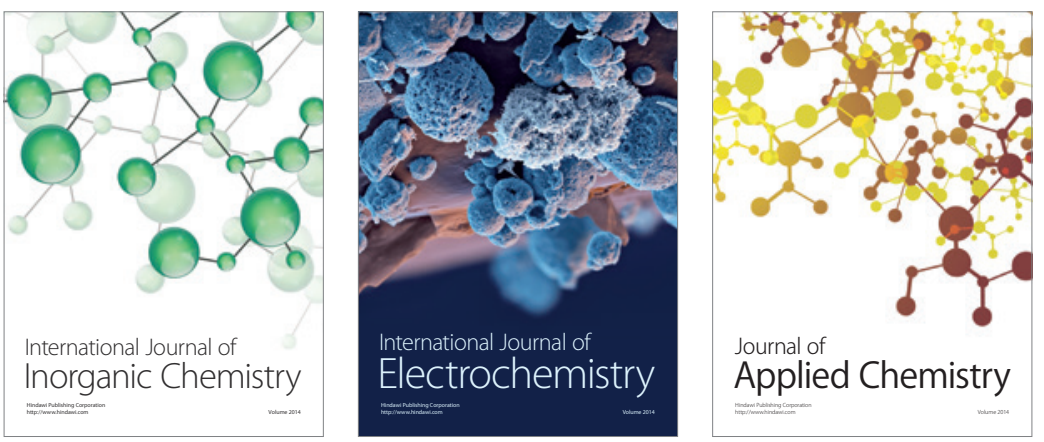

Journal of

Applied Chemistry
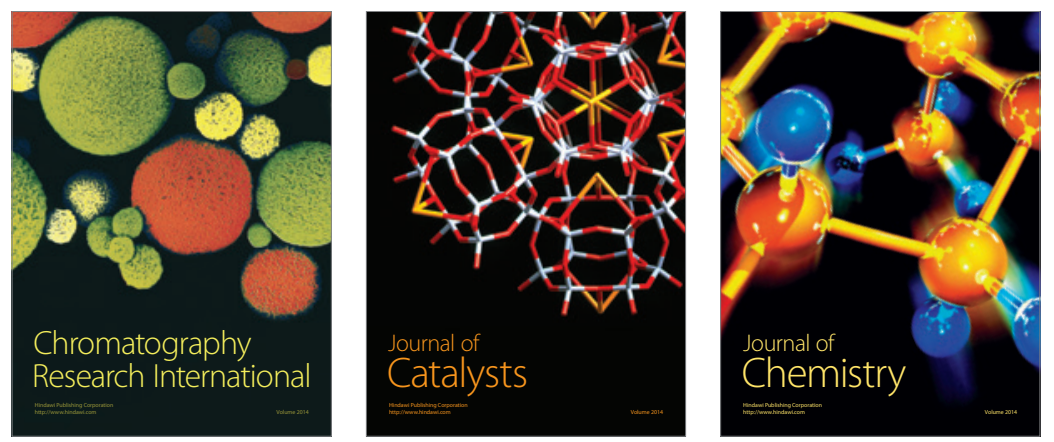
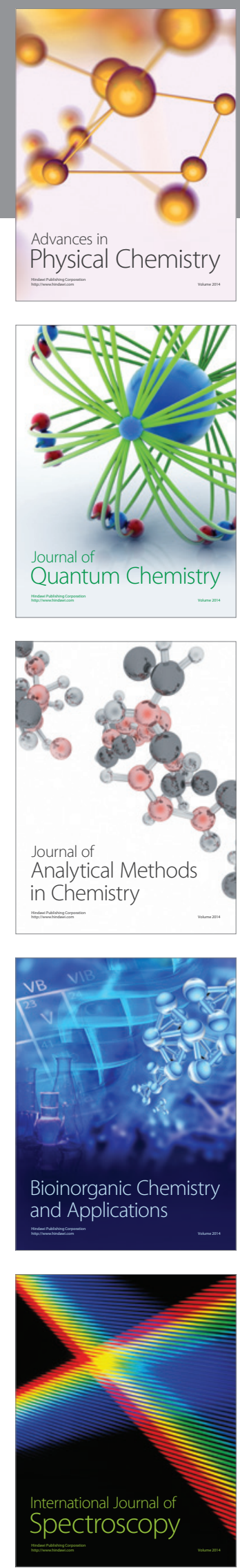